\title{
Isolation of ROS scavengers and development of its delivery system
}

\section{Roopam Raut` and Jessy Shaji}

Prin. K.M. Kundnani College of Pharmacy, Rambhau Salgaonkar Marg, Mumbai-400005, Maharashtra, India

\section{Article Info}

Article history

Received 10 May 2021

Revised 26 June 2021

Accepted 27 June 2021

Published online 30 June 2021

\section{Keywords}

Curcuminoids

Extraction

SMEDDS microemulsion

Antioxidant

\begin{abstract}
The present work was aimed at unlocking the pharmacologically active antioxidant molecules from the plant source and preserving their reactive oxygen species (ROS) scavenging activity in a well-planned environment with improved efficacy. Curcuminoids found in Curcuma species are multifaceted compounds containing curcumin, demethoxycurcumin, bisdemethoxycurcumin. In the present work, these were extracted efficiently from the rhizome of Curcuma longa L. with the help of statistical tools. The critical parameters like drug solvent ratio, extraction time were optimized. The identity of the compounds was confirmed with the help of instrumental techniques like HPTLC, FT-IR and HPLC. To improve solubility of sparingly soluble curcuminoids, without losing antioxidant properties was a challenge. Self-micro emulsifying delivery system (SMEDDS) was chosen as it has high drug loading capacity and is thermodynamically stable. The excipients were chosen based on solubility studies. The phase diagrams were constructed to define the formulation space. The developed delivery system was further evaluated for its permeability and ability to maintain antioxidant contents. The developed SMEDDS were able to achieve above desired quality attributes and can be marketed in future.
\end{abstract}

\section{Introduction}

For centuries, turmeric is being recognized and used worldwide in many different forms like curries, tea, drinks, sauce, cheese, chips and as a preservative and a coloring agent (Hewlings and Kalman, 2017). Turmeric, Curcuma longa (C. longa), is a perennial herb of Zingiberaceae family. It is grown in the various regions of world with tropical climate, but extensively in India and China, where it is part of not only food and medicine but sociocultural life. The rhizome portion of the plant is used predominantly as it can be dried, preserved for long time.

Turmeric powder, the powder of dried rhizomes, is about $60-70 \%$ carbohydrates, $6-13 \%$ water, $6-8 \%$ protein, $5-10 \%$ fat, $3-7 \%$ dietary minerals, $3-7 \%$ essential oils, $2-7 \%$ dietary fiber, and $1-6 \%$ yellow pigment. This golden yellow colour of the turmeric powder is due to curcuminoids, agent responsible for diverse health promoting effects (DiSilvestro et al., 2012). Curcumionids also possess therapeutic properties like anticancer (Liu and Ho, 2018), antiulcerative colitic (Lahiff and Alan, 2011), antiarthritic (Chandran and Goel, 2012), antidiabetic (Pivari et al., 2019), anti-alzheimeric (Ringman et al., 2005). To get maximum benefits, these curcuminoids are separated from turmeric and used. Since the turmeric rhizome contains multiple components, extraction of curcuminoids becomes tedious.

Curcuminoids is a mixture of three principal compounds: $77 \%$ of curcumin (curcumin I), 17\% of demethoxycurcumin (curcumin II), and $3 \%$ of bisdemethoxycurcumin (curcumin III) (Figure 1) (Srinivasan, 1953). These compounds are practically insoluble in

Corresponding author: Ms. Roopam Raut

Prin. K.M. Kundnani College of Pharmacy, Rambhau Salgaonkar Marg, Mumbai-400 005, India

E-mail: roopam4pharma@gmail.com

Tel.: +91-9820849384

Copyright () 2021 Ukaaz Publications. All rights reserved.

Email: ukaaz@yahoo.com; Website: www.ukaazpublications.com water at acidic and neutral $\mathrm{pH}$, and soluble in methanol, ethanol, dimethyl sulfoxide and acetone and unstable in alkaline $\mathrm{pH}$.

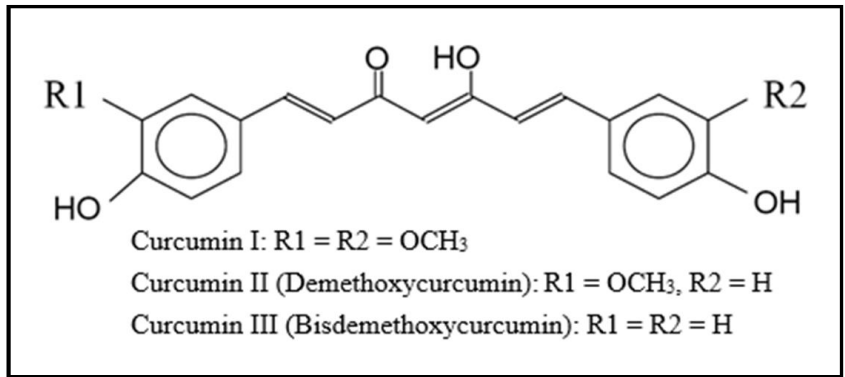

Figure 1: Chemical structure of curcumionoids.

The pharmacokinetics of curcumin, on oral administration was studied in Sprague-Dawley rats. Measurements of blood plasma levels and biliary excretion showed that curcumin was poorly absorbed from the gut. About $75 \%$ was excreted in the feces (Wahlstrom and Blennow, 1978). In this sense, it is comparable to class IV drugs of Biopharmaceutics Classification System.

To improve the bioavailability of curcumin, numerous approaches like complexation (Celebioglu and Uyar, 2020), novel particulate dosage forms (Chen et al., 2012; Acevedo-Guevara et al., 2018) were developed. The major disadvantage of these techniques is high cost, due to use of specialised excipients or manufacturing equipments. There is a need to develop stable dosage form with high drug loading efficiency and easy scalability at low cost. In the present work, curcuminoids were extracted to achieve maximum yield using statistical analysis and then were formulated into a self microemulsifying drug delivery system (SMEDDS). SMEDDS is a mixture of oil, surfactant and cosurfactant which on dilution with aqueous media forms transparent emulsion without the aid of mechanical equipment. 


\section{Materials and Methods}

\subsection{Chemicals and plant material}

Rhizomes of turmeric were collected from Palghar, Maharashtra, India. Vegetable oils were obtained from the local supermarket. Tween 80 and glycerol of LR grade were purchased from SD Fine Chem Industry. Ethyl oleate, hexane and propan-2-ol were from Loba Chemie. The standard curcumin (total curcuminoids $97 \%$ and curcumin $84 \%$ ) was purchased from Sigma-Aldrich Chemie GmbH. Crodamol GTCC was gifted by Croda India. Kollisolv PEG 400 was gifted by BASF, India. HPLC grade water was purchased from J.K. Labs, Thane, Maharashtra. All other solvents of AR grade and HPLC grade were purchased from E. Merck.

\subsection{Extraction of curcuminoids}

The fresh turmeric rhizomes were cleaned with water, sliced in thin pieces and dried in shade. The semi-dried material was further reduced to size less than 500 micron. The material was further dried in an oven to get the moisture content below $10 \% \mathrm{w} / \mathrm{w}$ and reduced to size less than 250 micron This turmeric powder was defatted using hexane. The solvent was removed with decantation and turmeric powder was dried at room temperature. This defatted turmeric powder was used for extraction.

In Soxhlet extraction, turmeric powder was placed in a "thimble" made of strong filter paper. The required quantity of methanol was added in distillation flask as a solvent. The turmeric powder and methanol were taken in the ratio of $1: 10$ to $1: 30$. The extraction was carried for 3 to $6 \mathrm{~h}$. Upon completion of the extraction, the solvent was separated from the extract under reduced pressure using rotary vacuum evaporator (Superfit Continental Pvt. Ltd, Mumbai, India). The resultant precipitate was washed twice with equal amount of cold propan-2-ol: hexane (1:1) to get purified curcuminoids. The weight of resultant curcuminoids was recorded.

\subsection{Identification of curcuminoids}

The standard and sample (extracted) curcuminoids were separately dissolved in methanol and optical density was recorded in the region 200 to $600 \mathrm{~nm}$ on UV-Vis Spectrophotometer (Evolution 300 ${ }^{\mathrm{TM}}$, Thermo Fisher Scientific, Hemel Hempstead, UK). HPTLC studies were performed using TLC Silica 60 F 254 plates. $1 \mathrm{mg} / \mathrm{ml}$ standard and sample curcuminoids solutions in acetone were applied in bands of $8 \mathrm{~mm}$ width. The plates were developed using solvents chloroform: methanol: formic acid in the ratio of 96:04: 01. CAMAG, Linomat 5, Switzerland, software was used for scanning the plates. The FTIR spectral studies for the standard and sample curcuminoids were carried out using potassium bromide as a substrate in the range of $400-4000 \mathrm{~cm}^{-1}$ on Bruker Alpha T (Bruker Optik GmbH, Ettlingen, Germany). The HPLC analysis was carried out on Agilent 1260 Infinity II (Agilent Technologies Deutschland GmbH, Waldbronn, Germany) equipped with UV/Vis Detector. Mobile phase used for analysis was composed of acetonitrile: $0.1 \%$ orthophosphoric acid in HPLC water $(35: 65 \% \mathrm{v} / \mathrm{v})$. The standard and sample curcuminoids were dissolved in methanol. Column Inertsil ODS-3V, 150*4.6 mm, 5 $\mu \mathrm{m}$ (GL Sciences Inc, Tokyo, Japan) was used for analysis. The detection was carried out at wavelength of $420 \mathrm{~nm}$.
2.4 Formulation development for curcuminoids

\subsubsection{Solubility study for curcuminoids}

Curcuminoids solubility studies in various oils, surfactants, and co-surfactants were carried out. In a glass vial, $5 \mathrm{~g}$ of vehicle were taken to which curcuminoids were added in increments of $10 \mathrm{mg}$. The samples were stirred. The procedure was repeated till the solution was saturated and undissolved curcuminoids were visible. Each sample was stirred at room temperature for $24 \mathrm{~h}$. After reaching equilibrium, each vial was centrifuged at $7000 \mathrm{rpm}$ for $20 \mathrm{~min}$. The supernatant was filtered through a $0.45 \mu \mathrm{m}$ syringe filter membrane and diluted with methanol for quantification by UV-Visible spectrophotometer.

\subsubsection{Construction of phase diagrams}

Tween-80 and Kollisolv PEG 400 were mixed in the ratio 1:1, 2:1 and 4:1 to form surfactant mix (Smix). Oil (Crodamol GTCC) and Smix were mixed in the ratio of 1:9 to 9:1. Water was added in each test tube dropwise and mixed using vortex mixer. The observations were recorded. The clear transparent homogenous solution was considered as a microemulsion and phase diagrams were prepared.

\subsubsection{Preparation of curcuminoids SMEDDS}

On the basis of ternary phase diagrams SMEDDS components were selected for drug incorporation. Three batches F1 (10\% oil and 90\% Smix) F2 (12.5\% oil and $87.5 \%$ Smix) and F3 (16.67\% oil and $83.33 \%$ Smix) were prepared. The curcuminoids were added to it at the concentration of $2 \% \mathrm{w} / \mathrm{w}$ and stirred at room temperature for $30 \mathrm{~min}$.

\subsection{Characterization of curcuminoid SMEDDS}

\subsubsection{Clarity and particle size}

After preparation, the SMEDDS were observed visually. Further, the SMEDDS were diluted with $250 \mathrm{ml}$ of $0.1 \mathrm{~N} \mathrm{HCl}$ and stored at $37^{\circ} \mathrm{C}$ to see the effect of dilution. The resultant solutions were examined spectrophotometrically at wavelength of $652 \mathrm{~nm}$ and per cent transmittance were recorded. Formulations F1 and F2 were further studied as they showed high transmittance. F1 and F2 were diluted 250 times and then droplets size was measured using Nano Sight NS500 (Malvern Instruments, Amesbury, UK).

\subsubsection{In vitro dissolution studies}

The SMEDDS formulations were filled in hard gelatin capsules (size 00 ) and introduced in dissolution jars of USP dissolution apparatus II (EDT-208Lx, Electrolab India Pvt Ltd, Mumbai, India). Each jar contained $500 \mathrm{ml}$ of $0.1 \mathrm{~N} \mathrm{HCl}$ maintained at $37^{\circ} \mathrm{C}$ and stirred at 50 rpm. The aliquots were taken at 0,10,20.30, 40 and $60 \mathrm{~min}$ and replaced with equal quantity of dissolution media. The aliquots were filtered through $0.22 \mu \mathrm{m}$ syringe filter and curcuminoids content were analysed by HPLC. The determination was performed on a C18 column $(4.6 \mathrm{~mm} \times 150 \mathrm{~mm}, 5 \mu \mathrm{m}$, Intersil ODS $)$ with a mobile phase of acetonitrile: $0.1 \%$ orthophosphoric acid $(35: 65)$. The analysis was carried out at the wavelength of $420 \mathrm{~nm}$. The graphs were plotted for $\%$ cumulative drug release Vs time. The dissolution profile was compared with dissolution profile of capsule filled with equivalent quantity of the drug suspended in phosphate buffer of $\mathrm{pH} 5.5$. 


\subsubsection{In vitro permeability studies}

The small intestine of overnight fasted chick (male, 0.5-0.6 kg) was immediately collected after slaughtering. The small intestine was washed with phosphate buffer solution and was cut into $6.0 \pm 0.5$ $\mathrm{cm}$ pieces. One end of intestine was tied with the thread. Curcuminoids and curcuminoid SMEDDS equivalent to $4 \mathrm{mg}$ of the drug were dispersed in water and then carefully introduced into the intestine using blunt needle and syringe. The other end of the intestine was closed. Each sac was placed in a glass tube containing $20 \mathrm{ml}$ of equal mixture of ethanol and phosphate buffer $\mathrm{pH} 5.5$. The entire system was maintained at $37^{\circ} \mathrm{C}$ and was mounted on rotary shaker. Aliquots were withdrawn from outside the sac and the medium was replaced by fresh medium. The concentration of curcuminoids which were able to cross the membrane was measured by HPLC.

\subsubsection{Antioxidant activity (AA) stability studies}

\subsubsection{DPPH free radical scavenging activity studies}

Curcuminoids and curcuminoids SMEDDS were diluted with methanol and mixed with $2 \mathrm{ml}$ of $0.004 \%$ DPPH solution. The solution was stored in the dark for $30 \mathrm{~min}$ and absorbance were recorded at $517 \mathrm{~nm}$. The per cent inhibitions in free radical scavenging activities were calculated by following formula:

$\%$ Inhibition $=(($ Abs of control - Abs of sample $) / \mathrm{Abs}$ of control $) \times$ $100 \ldots$ (Equation 1).

\subsubsection{Hydrogen peroxide scavenging activity studies}

Curcuminoids and curcuminoids SMEDDS were diluted with ethanol and mixed with $2 \mathrm{ml}$ of $20 \mathrm{mM}$ solution of hydrogen peroxide in phosphate buffer ( $\mathrm{pH} 7.4$ ). After $10 \mathrm{~min}$, the absorbance was measured at $230 \mathrm{~nm}$ using Spectrophotometer. The per cent inhibitions in hydrogen peroxide scavenging activities were calculated by following formula:

$\%$ Inhibition $=(($ Abs of control - Abs of sample $) / \mathrm{Abs}$ of control $) \times$ $100 \ldots$ (Equation 2)

Curcuminoid and curcuminoids SMEDDS were further stored at $60^{\circ} \mathrm{C}$ in glass vials for 14 days. AA of the curcuminoids and curcuminoids SMEDDS stored at $60^{\circ} \mathrm{C}$ were compared with initial AA. The per cent reduction in antioxidant activity inhibition was calculated by the following equation:

$\%$ Reduction $=\left(\left(\mathrm{AA}_{\text {initial }}-\mathrm{AA}_{\text {on } 14 \text { days at } 60^{\circ} \mathrm{C}}\right) / \mathrm{AA}_{\text {initial }}\right) \times 100 \ldots \ldots \ldots \ldots$ (Equation 3)

\subsubsection{Statistical analysis}

The experiments were performed in triplicate. The data were recorded as mean \pm standard deviation and analysed by Microsoft office Home and Student edition of 2016. Significant differences between means for unformulated curcuminoids and curcuminoids SMEDDS were determined by student $\mathrm{t}$ test, and $p<0.05$ was regarded as significant, and $p<0.01$ was very significant.

\section{Results}

\subsection{Extraction of curcuminoids}

The extraction conditions were optimized by using statistical software Design Expert ( 8.0 software (Stat-Ease, Inc., Minneapolis). Central Composite design with three center point at alpha value equal to 1 was used in the study. Drug solvent ratio and time were taken as independent variables and \% yield as a dependent variable. Total 11 batches were prepared (Table 1).

Table 1: Design matrix for extraction of curcuminoids

\begin{tabular}{|l|c|c|c|c|}
\hline Std & Run & $\begin{array}{c}\text { Factor 1 } \\
\text { A:Aerb } \\
\text { solvent } \\
\text { ratio) }\end{array}$ & $\begin{array}{c}\text { Factor } \text { 2 } \\
\text { (Time) }\end{array}$ & Response \\
\hline 11 & 1 & 22.5 & 4.5 & yield \\
\hline 7 & 2 & 22.5 & 3 & 5.63 \\
\hline 8 & 3 & 22.5 & 6 & 6.1 \\
\hline 4 & 4 & 30 & 6 & 6.12 \\
\hline 1 & 5 & 15 & 3 & 5.11 \\
\hline 6 & 6 & 30 & 4.5 & 6.02 \\
\hline 5 & 7 & 15 & 4.5 & 5.25 \\
\hline 3 & 8 & 15 & 6 & 5.56 \\
\hline 10 & 9 & 22.5 & 4.5 & 5.7 \\
\hline 9 & 10 & 22.5 & 4.5 & 5.75 \\
\hline 2 & 11 & 30 & 3 & 5.88 \\
\hline
\end{tabular}

The central composite model used in the study was found to be significant with f-value of 46.67 . There was only a $0.03 \%$ chance that an f-value, this large could occur due to noise. The response surface plot showed the effect of solvent quantity and time on yield (Figure 2). The optimized equation for the yield was:

Yield $=+2.79487+0.191877 \mathrm{~A}-0.001725 \mathrm{~B}-0.004667 \mathrm{~A}$

* B-0.002760 $\mathrm{A}^{2}+0.026550 \mathrm{~B}^{2} \ldots \ldots$. (Equation 4)

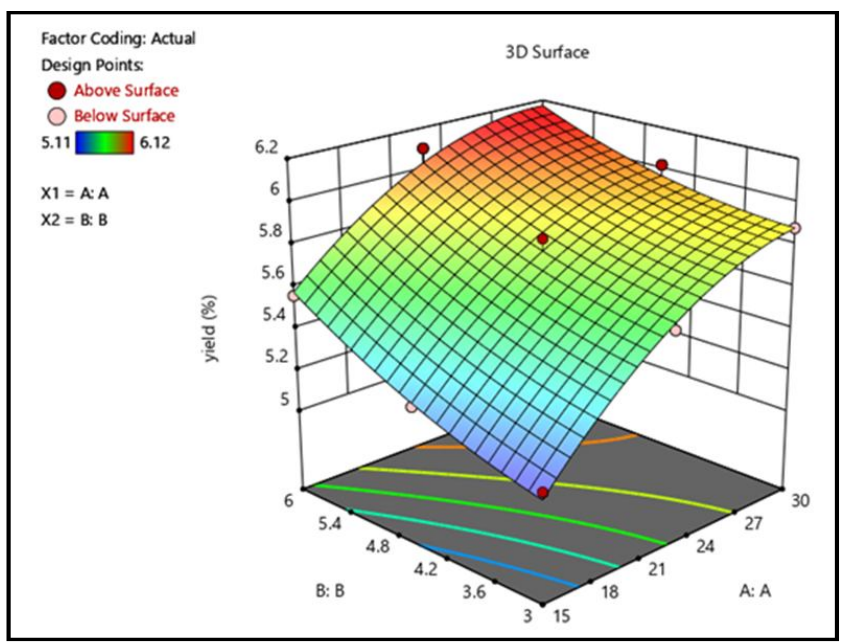

Figure 2: Response surface plot showing the effect of A:A (Herb: solvent ratio) and B:B (Time) on \% yield.

\subsection{Identification of curcuminoids}

UV-VIS spectrum of methanolic solution of standard and sample curcuminoids matched with each other and showed maximum absorbance at a wavelength of $420 \mathrm{~nm}$. In HPTLC studies, 
bisdemethoxycurcumin, desmethoxycurcumin and curcumin were detected at $\mathrm{Rf}$ of $0.203,0.272$ and 0.444 , respectively (Figure 3) (Paramasivam, 2009). The percentage of curcumin and total curcuminoids were found to be $78.54 \%$ and $95.45 \%$, respectively. FT-IR spectra of standard and sample curcuminoids (Figure 4) showed vibrations due to various chemical groups like $-\mathrm{OH}$ vibrations at $3508 \mathrm{~cm}^{-1}$, C-H aromatic stretching vibration at $3057 \mathrm{~cm}^{-1}$, -CH3- asymmetric stretching at $2947 \mathrm{~cm}^{-1},-\mathrm{C}=\mathrm{O}$ stretching at 1627,1602 $\mathrm{cm}^{-1},-\mathrm{C}=\mathrm{C}$ aliphatic stretching at $1510 \mathrm{~cm}^{-1},-\mathrm{C}=\mathrm{C}$ aromatic stretching at $1456 \mathrm{~cm}^{-1}$ and phenol group at $1281 \mathrm{~cm}^{-1}$. HPLC chromatograms (Figure 5) showed 3 distinct peaks corresponding to bisdemethoxycurcumin, demethoxycurcumin and curcumin at RT of 27.97, 32.41 and 37.39 for standard and sample curcuminoids. The total curcuminoids content was $98.3 \%$ and curcumin content was $81.8 \%$.

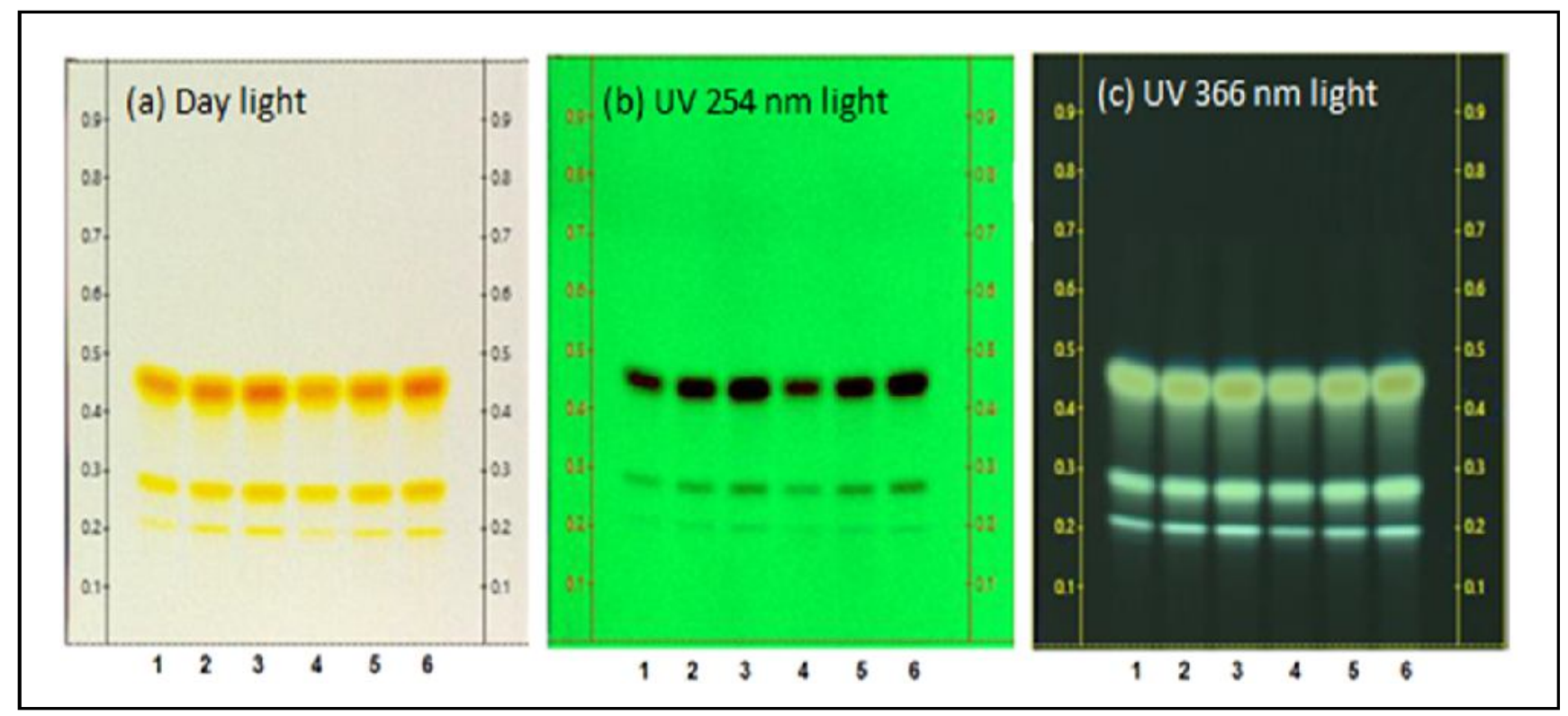

Figure 3: HPTLC chromatograms of curcuminoids (Track 1-3 of Standard and Track 4-6 of sample curcuminoids).

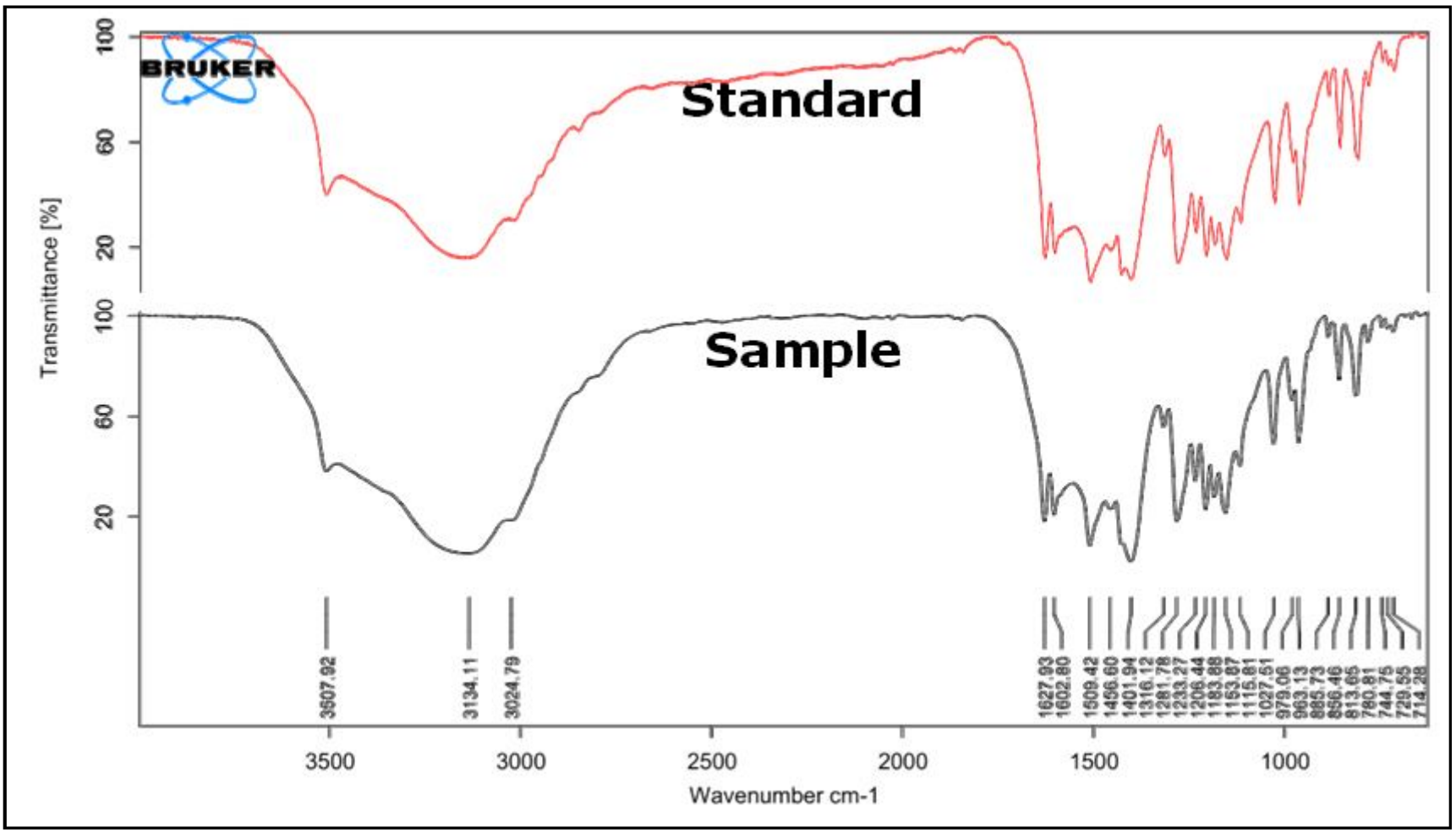

Figure 4: FT-IR spectra of standard and sample curcuminoids. 


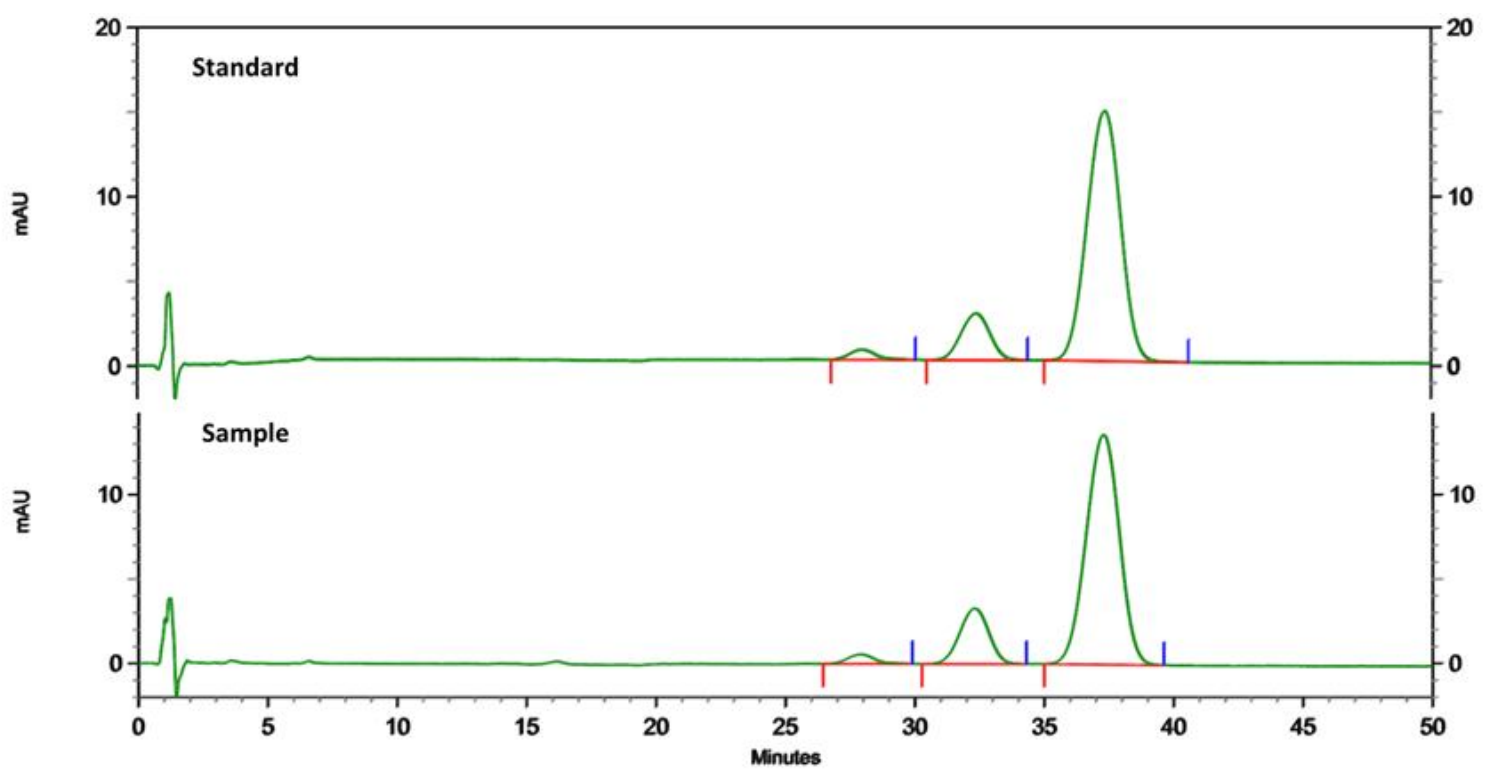

Figure 5: HPLC chromatograms of standard and sample curcuminoids.

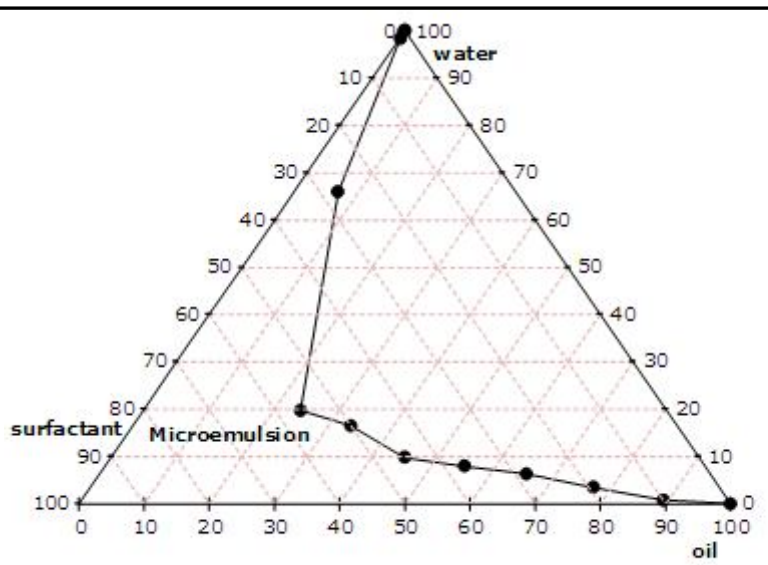

(a) Surfactant : Cosurfactant (Km) 1:1

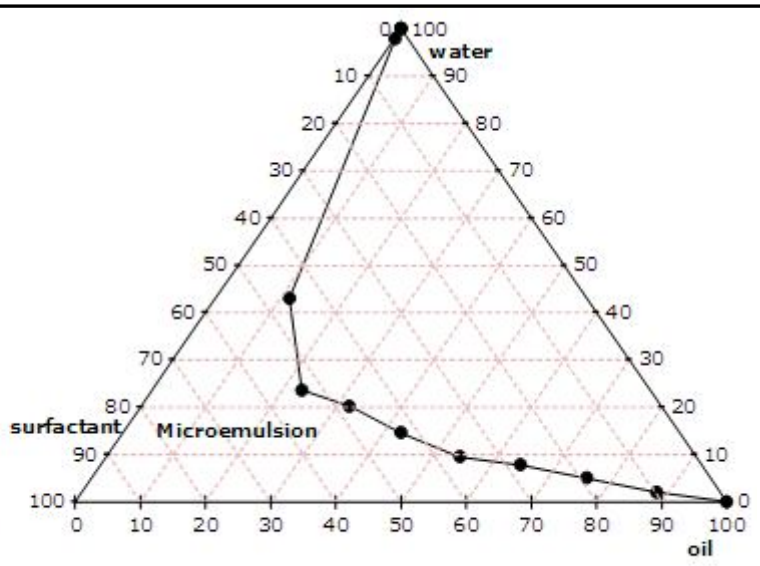

(b) Surfactant: Cosurfactant (Km) 2:1

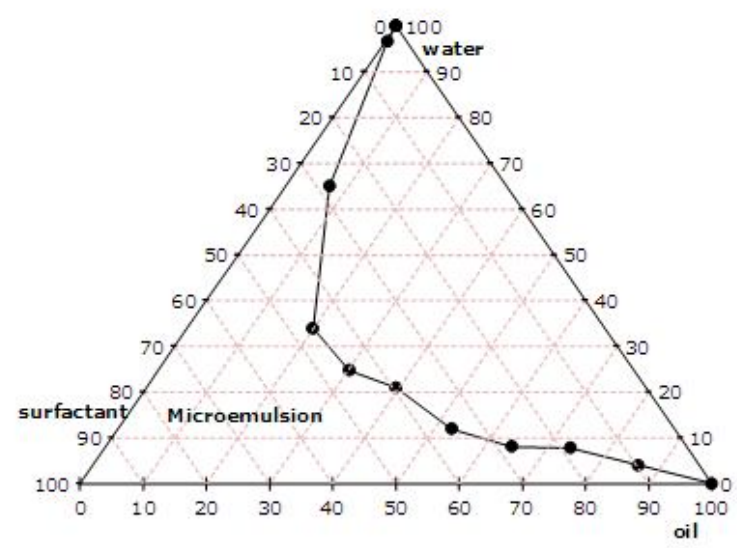

(c) Surfactant : Cosurfactant (Km) 4:1

Figure 6: Pseudoternary phase diagrams indicating microemulsion regions for systems containing crodamol GTCC (oil), Tween 80: Kollisolv PEG 400 and water. 


\subsection{Formulation development for curcuminoids}

Curcuminoids showed up to $0.1 \% \mathrm{w} / \mathrm{w}$ solubility in sunflower oil, soybean oil, sesame oil and isopropyl myristate. Ethyl oleate, glycerine, polyglycerol oleate, span 80 showed intermediate solubility. Highest solubility was found in crodamol GTCC, kollisolv PEG 400 and tween 80.

Based on solubility studies excipients chosen for further studies were crodamol GTCC (oil), tween 80 (surfactant) and kollisolv PEG 400 (cosurfactant). The pseudo ternary phase diagrams showing microemulsion regions were constructed (Figure 6).

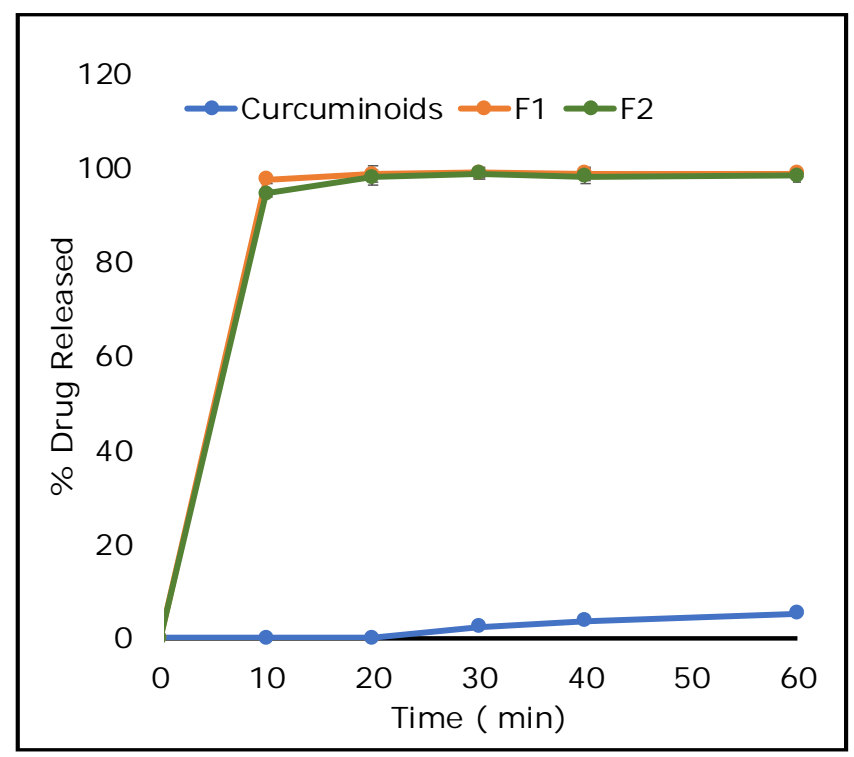

Figure 7: In vitro release profiles of curcuminoids and curcuminoid SMEDDS.

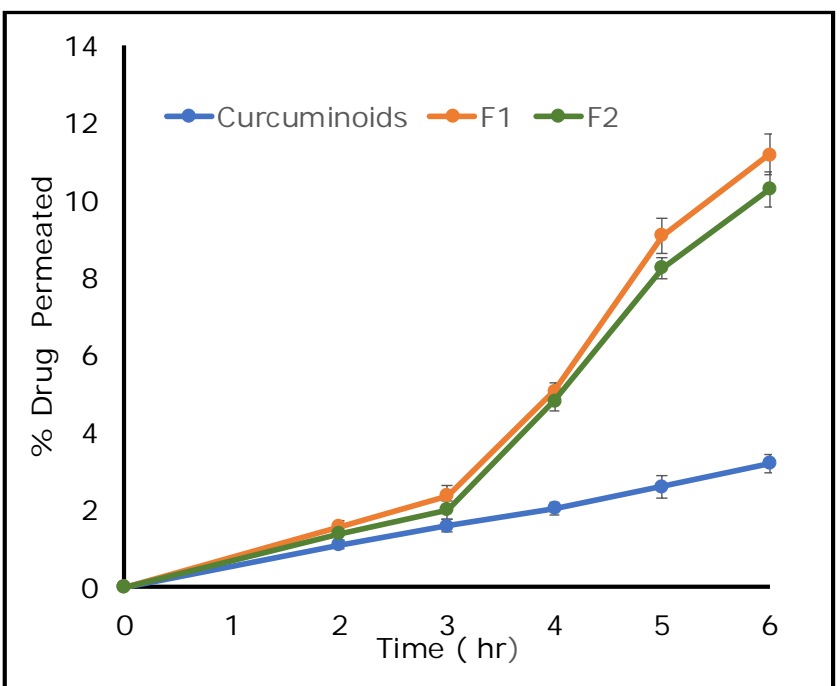

Figure 8: In vitro permeation profiles of curcuminoids and curcuminoid SMEDDS.

\subsection{Characterization of curcuminoids SMEDDS}

Among 3 selected formulations, F3 showed least \% transmittance $91.54 \pm 0$. Batch F1 and F2 showed good \% transmittance $(99.35 \pm$ 0.30 and $97.69 \pm 0.60$, respectively), hence, further studied for droplet size analysis. Though F1 had smaller globule size (148 \pm $3.51 \mathrm{~nm})$ as compared to F2 $(171 \pm 3.05 \mathrm{~nm})$, both batches showed globule size less than $200 \mathrm{~nm}$. The dissolution profiles of curcuminoid SMEDDS F1 and F2 showed significantly high release as compared to curcuminoids (Figure 7). In vitro permeability studies showed 3.49 and 3.21 times increase in amount of drug permeated as compared non-formulated curcuminoids (Figure 8). Results of DPPH antioxidant stability studies showed that \% reduction in antioxidant activity after 14 days on storage at $60^{\circ} \mathrm{C}$ were $2.23 \pm 0.13,2.58 \pm 0.36$ and $2.65 \pm 047$ for unformulated curcuminoids, SMEDDS F1 and F2, respectively. Hydrogen peroxide scavenging activity stability studies showed that $\%$ reduction in antioxidant activity due to thermal effect were $2.86 \pm 0.09,2.66 \pm$ 0.36 and $2.73 \pm 0.05$ for unformulated curcuminoids, SMEDDS F1 and $\mathrm{F} 2$, respectively.

\section{Discussion}

\subsection{Extraction and identification of curcuminoids}

In the present study, oil depleted turmeric was extracted with methanol, as curcuminoids have high solubility in methanol (Verghese, 1993). Though, various advanced techniques are available for extraction, solvent extraction method was followed as it is a simple method to work on laboratory scale. It was observed that as herb solvent ratio and time for extraction was increased, yield increased. To extract maximum quantity of curcuminoids using minimum solvent and minimum time was the aim of the optimization. When herb solvent ratio of was set at 1:25 and extraction was carried out for $4 \mathrm{~h}$ and $50 \mathrm{~min}, 5.76 \pm 0.16 \%$ yield was obtained as against predicted yield of $5.91 \%$. This close agreement of values, confirmed suitability of design. The instrumental studies carried out using UV, HPTLC, FT-IR and HPLC confirmed identity and purity of curcuminoids.

\subsection{Formulation development for curcuminoids}

Self-microemulsifying drug delivery system (SMEDDS) has recently received attention due to its ability to enhance solubility and permeability. It is a useful technique for orally administered, poorly water-soluble drugs. SMEDDS (Gursoy and Benita, 2004) is a mixture of oil, surfactant, co-surfactant and on dilution with gastrointestinal fluid under the influence of gastric motility it forms a microemulsion (Rajpoot et al., 2020; Hintzen et al., 2013; Vithani et al., 2019). Being isotropic in nature, it forms clear solution which is well accepted by patients. Liquid SMEDDS can be suitably colored and flavored for oral administration or can be filled in hard/soft capsules. Hence, it has convenience of dose adjustment and can be given as a unit or bulk dosage form. Tween 80 and PEG 400, the excipients with high curcuminoid solubility are high HLB nonionic surfactant and cosurfactant. Because of non-ionic in nature, it is more stable to presence of ions, change in $\mathrm{pH}$ and due to high HLB it easily forms o/w emulsion. Hence, the choice of excipients was in agreement with the aim of preparing oral formulation. It was observed that as when $\mathrm{Km}$ ratio was increased from 1:1 to 4:1, microemulsion region increased. Hence, $\mathrm{Km}$ ratio 4:1, was used in further studies.

\subsection{Characterization of curcuminoids SMEDDS}

Poor transmittance of F3 may be due to poor entrapment efficiency of the oil and Smix. For formulations F1 and F2, globule size was in 
colloidal range and the formulations were able to withstand dilution effect. Solubilization of drug in oil and surfactant mix had helped to achieve $100 \%$ drug release in in vitro dissolution studies. Small globules of microemulsion provided effective environment for lipophilic curcuminoids to easily cross the membrane and formulations showed high permeability (Nipun and Islam, 2014). ROS is the term used for various reactive molecules and free radicals. Higher levels of ROS are formed during stress and can lead to cancer, diabetes mellitus, cardiovascular diseases, atherosclerosis, arthritis, etc. Curcuminoids regulate ROS production which helps in cellular homeostasis. Free radical scavenging activities for curcuminoids and curcuminoids SMEDDS were measured using DPPH $(2,2$ diphenyl-1-picryl hydrazyl) reagent method introduced by Blois (1958) with suitable modifications. DPPH free radical scavenging assay is an easy, fast and precise method to determine antioxidant activity. The odd electron of nitrogen atom in DPPH is reduced by free radical to the corresponding hydrazine. Correspondingly purple color of DPPH is lost. The hydrogen peroxide scavenging activity was carried out based on the principle introduced by Ruch et al. (1989). The removal of hydrogen peroxide is very important for antioxidant defense of cells, as it causes oxidation of proteins, lipids and DNA. As antioxidant reacts with hydrogen peroxide, its concentration decreases and so is the absorbance. The percent reduction in DPPH free radical scavenging activity were not significant as compared to unformulated curcuminoids for both F1 and F2 with p value 0.33 and 0.17 , respectively. Similar results were obtained for hydrogen peroxide scavenging activity with $p$ values 0.36 and 0.27 . This confirmed that curcuminoids were stable in SMEDDS and retained their antioxidant property.

\section{Conclusion}

This article describes a method for the extraction of curcuminoids by solvent extraction. The experimental design approach using response surface methodology was successfully applied to optimize solvent quantity and time. The isolated curcuminoids were identified through HPTLC, FT-IR and HPLC. Though, curcuminoids are therapeutically versatile, poor bioavailability demands higher dose, which is not convenient for the patient. The selected excipients were able to give preparations which spontaneously formed stable microemulsion with fine particle size. By incorporating curcuminoids in SMEDDS, inherent short comings of curcuminoids, viz., low solubility and poor dissolution were effectively addressed while still preserving antioxidant activities. More than 3 times increase in the amount of drug permeated by curcuminoids SMEDDS as compared non-formulated curcuminoids was observed. This indicates that formulation with low dose will be effective and there will be high patient compliance. The formulations were stable to heat and retained antioxidant activity. The formulations can be further studied in animals and human beings.

\section{Acknowledgements}

We acknowledge the facility under DST-FIST Funding (Letter SR/ FST/College-264 dated 18th November, 2015). Financial assistance for experimental work was provided by University of Mumbai Ref.: Research Project No.: 512 (APD/ICD/ 2018-19/593 of 2018 dated $16^{\text {th }}$ March 2019).

\section{Conflict of interest}

The authors declare that there are no conflicts of interest relevant to this article.

\section{References}

Acevedo Guevara, L.; Nieto Suaza, L.; Sanchez, L.T.; Pinzon, M.I. and Villa, C.C. (2018). Development of native and modified banana starch nanoparticles as vehicles for curcumin. Int. J. Biol. Macromol., 111:498-504.

Blois, M.S. (1958). Antioxidant determinations by the use of a stable free radical. Nature, 181(4617):1199-1200.

Celebioglu, A. and Uyar, T. (2020). Fast-dissolving antioxidant curcumin/ cyclodextrin inclusion complex electrospun nanofibrous webs. Food Chem., 317:1263-1297.

Chandran, B. and Goel, A. (2012). A randomized, pilot study to assess the efficacy and safety of curcumin in patients with active rheumatoid arthritis. Phytother. Res., 26(11):1719-1725.

Chen, Y.; Wu, Q.; Zhang, Z.; Yuan, L.; Liu, X. and Zhou, L. (2012). Preparation of curcumin-loaded liposomes and evaluation of their skin permeation and pharmacodynamics. Molecules, 17(5):5972-5987.

DiSilvestro, R.A.; Joseph, E.; Zhao, S. and Bomser, J. (2012). Diverse effects of a low dose supplement of lipidated curcumin in healthy middleaged people. Nutriti. J., 11:79.

Gursoy, R.N. and Benita, S. (2004). Self-emulsifying drug delivery systems (SEDDS) for improved oral delivery of lipophilic drugs. Biomed. Pharmacother., 58(3):173-182.

Hewlings, S.J. and Kalman, D.S. (2017). Curcumin: A review of its effects on human health. Foods, 6(10):92.

Hintzen, F.; Laffleur, F.; Sarti, F.; Müller, C. and Bernkop-Schnürch, A. (2013). In vitro and ex vivo evaluation of an intestinal permeation enhancing self-microemulsifying drug delivery system (SMEDDS). J. Drug Deliv. Sci. Technol., 23(3):261-267.

Lahiff, C. and Moss, A.C. (2011). Curcumin for clinical and endoscopic remission in ulcerative colitis. Inflamm. Bowel. Dis., 17(7):E66.

Liu, H.T. and Ho, Y.S. (2018). Anticancer effect of curcumin on breast cancer and stem cells. Food. Sci. Hum. Wellness, 7(2):134-137.

Nipun, T.S. and Islam, S.A. (2014). SEDDS of gliclazide: Preparation and characterization by in vitro, ex vivo and in vivo techniques. Saudi Pharm. J., 22(4):343-348.

Paramasivam, M.; Poi, R.; Banerjee, H. and Bandyopadhyay, A. (2009). Highperformance thin layer chromatographic method for quantitative determination of curcuminoids in Curcuma longa germplasm. Food. Chem., 113(2):640-644.

Pivari, F.; Mingione, A.; Brasacchio, C. and Soldati, L. (2019). Curcumin and type 2 diabetes mellitus: Prevention and treatment. Nutrients, 11(8): 1837.

Rajpoot, K.; Tekade, M.; Pandey, V.; Nagaraja, S.; Youngren-Ortiz, S.R. and Tekade, R.K. (2020). Self-microemulsifying drug-delivery system: Ongoing challenges and future ahead. In: Drug delivery systems (ed. Tekade, R.K.), Academic Press, Cambridge, MA, USA, pp:393-454. 
Ringman, J.M.; Frautschy, S.A.; Cole, G.M.; Masterman, D.L. and Cummings, J.L. (2005). A potential role of the curry spice curcumin in Alzheimer's disease. Curr. Alzheimer Res., 2(2):131-136.

Ruch, R. J.; Cheng, S. J. and Klaunig, J. E. (1989). Prevention of cytotoxicity and inhibition of intercellular communication by antioxidant catechins isolated from Chinese green tea. Carcinogenesis, 10(6): $1003-1008$

Srinivasan, K.R. (1953). A chromatographic study of the curcuminoids in Curcuma longa L. Journal of Pharm. Pharmacol., 5(1):448-457.
Verghese, J. (1993). Isolation of curcumin from Curcuma longa L. rhizome. Flavour Fragr. J., 8(6):315-319.

Vithani, K.; Jannin, V.; Pouton, C.W. and Boyd, B.J. (2019). Colloidal aspects of dispersion and digestion of self-dispersing lipid-based formulations for poorly water-soluble drugs. Adv. Drug Deliv. Rev., 142:16-34.

Wahlstrom, B. and Blennow, G. (1978). A study on the fate of curcumin in the rat. Acta Pharmacol. Toxicol., 43(2):86-92. 